\title{
Gerontology
}

\section{Subjective Memory in a National Sample: Predicting Psychological Well-Being}

\author{
Jacqueline A. Mogle ${ }^{a}$ Nikki Hill ${ }^{b}$ Caroline McDermott ${ }^{b}$ \\ ${ }^{a}$ Center for Healthy Aging, College of Nursing, and ${ }^{b}$ College of Nursing, The Pennsylvania State University, \\ University Park, PA, USA
}

\section{Keywords}

Subjective memory · Subjective cognition - Well-being .

Depressive symptoms · Life satisfaction

\begin{abstract}
Background: Individual perception of memory performance (i.e., subjective memory) is assessed using a variety of approaches. This article focuses on 2 such approaches: (1) selfcomparison assessments that attempt to capture changes in memory ability over a period of time and (2) age-anchored comparisons that assess how an individual perceives their memory in relation to others their age. These different types of assessment may relate to psychological well-being differently due to the underlying mechanisms of assessment. $\mathbf{O b}$ jective: The purpose of these analyses is to examine 2 measures of subjective memory (i.e., a self-comparison measure and an age-anchored comparison measure) as predictors of psychological well-being among adults in mid- and late life. Methods: Participants $(n=3,434)$ in the Midlife in the United States Study completed measures of subjective memory, depressive affect, and life satisfaction. Structural equation modeling was used to examine whether the self-comparison and age-anchored comparison measures had differential predictive utility regarding psychological well-being. $\boldsymbol{R e}$ -
\end{abstract}

\section{KARGER}

(C) 2017 S. Karger AG, Basel

E-Mail karger@karger.com

www.karger.com/ger sults: Higher age-anchored comparison ratings were related to higher life satisfaction scores. There was a significant interaction between the 2 items such that individuals with lower ratings on both subjective memory measures had the poorest outcomes. Additionally, age-anchored comparisons interacted with age: older adults had the poorest outcomes when they reported poorer age-anchored comparisons. Conclusion: These findings highlight the importance of precise measurement in the consideration of subjective memory. How an individual was asked to rate his or her perception of memory influenced the relationships between subjective memory and psychological well-being. This study contributes valuable insight into the importance of the assessment models of subjective memory.

(c) 2017 S. Karger AG, Basel

\section{Introduction}

Subjective memory is a term used to indicate how individuals interpret, feel, or think about their own memory - in other words, one's perceptions regarding memory performance [1]. Studies examining the relationship between subjective memory and concurrent objective memory performance have been decidedly mixed, with some

Jacqueline A. Mogle

Center for Healthy Aging, College of Nursing

The Pennsylvania State University

201 Nursing Sciences Building, University Park, PA 16802 (USA)

E-Mail jam935@psu.edu 
reporting weak positive associations and others no association [e.g., 1, 2]. Subjective memory is influenced by a multitude of factors beyond actual memory performance. Regardless of objective memory performance, poorer subjective memory is frequently linked to increased psychological distress [3-5]; however, the literature on this topic has focused on the relationship of subjective memory to depressive symptoms without considering other aspects of psychological well-being (e.g., life satisfaction [6]). Additionally, no studies have examined whether different approaches to the assessment of subjective memory differentially predict psychological well-being. There is great variability in current subjective memory measures. In their recent review, Rabin et al. [7] urge caution in comparing findings relevant to subjective memory across studies due to the substantial heterogeneity of measures. There is an identified need to interpret findings that associate subjective memory and outcomes with specificity: item construction matters.

In response, this study compares 2 different measures of subjective memory to simultaneously predict psychological well-being, including age as a potential moderator of these relationships, in a national sample of adults.

\section{Subjective Memory Assessment}

Subjective memory, or an individual's perceived memory performance, is influenced by a combination of ability judgments and memory concerns [8]. Ability judgments are based on exposure to memory problems that affect our perceptions of our memory. For example, forgetting to pick up milk from the store may cause us to reflect on the frequency of such an event and negatively evaluate our memory performance. Memory concerns are based on fears or worries about memory problems (and decline) including perceiving one's memory functioning as poorer than peers'. In general, ability judgments are thought to stem from concrete events, while memory concerns are thought to be influenced by beliefs about memory including, but not limited to, aging-related stereotypes [8].

Ability judgments and memory concerns are represented in 2 common approaches to subjective memory assessment: (1) self-comparisons and (2) age-anchored comparisons [9]. Self-comparisons measure subjective memory by asking individuals to compare their present memory to their memory in the past (i.e., "Is your memory as good as it was 5 years ago?"). To answer these questions, respondents must evaluate and compare their past and current memory ability, rather than concerns about memory. In contrast, age-anchored comparisons assess

Subjective Memory in a National Sample subjective memory by asking individuals to compare their present memory to their peers' (i.e., "Do you feel you have more memory problems than others your age?"). Age-anchored comparisons are viewed as a social threat activating an individual's concerns about their memory [10]. When making age-anchored comparisons, respondents frequently react by evaluating themselves in a more complimentary way [11], a phenomenon referred to as the better-than-average effect [12].

\section{Subjective Memory and Psychological Well-Being}

Psychological well-being is a multidimensional construct represented by both negative facets, such as depressive symptoms, as well as positive facets, such as life satisfaction [13]. Perceptions of memory play a key role in the psychological well-being of adults. Indeed, multiple studies have demonstrated associations between poorer subjective memory ratings and greater depressive symptoms as well as higher levels of stress, which may in turn exacerbate memory-related problems $[6,14,15]$. This relationship may be more pronounced for older adults. Perceiving one's memory as poor or declining is a threat to aging well: perceptions of memory problems precipitate emotional distress and worry about dementia or loss of independence [16, 17]. Coping may also be particularly difficult for this age group, as cognitive decline (aging or dementia related) may be considered inevitable.

With respect to positive facets of psychological wellbeing, Toffalini et al. [18] found a significant positive relationship between subjective memory and overall wellbeing in older adults. However, this relationship is far less evidenced in the literature. It is important to consider how an individual's memory perceptions impact their psychological well-being both in terms of increases in negative symptoms, such as depressive symptoms, as well as decreases in positive aspects, such as life satisfaction [19].

As described above, self-comparisons and age-anchored comparisons rely on 2 different intrapersonal strategies for memory evaluation which may reflect their corresponding relationships to psychological well-being. Age-anchored comparisons activate social threat mechanisms but may not be related to actual performance decrements. In contrast, self-comparisons reflect perceived decline in memory performance indicating poorer functioning more broadly [8]. However, it remains unclear whether these different mechanisms will result in differential relationships to positive and negative aspects of psychological well-being. The current study will explore these relationships in more depth.

Gerontology 2017;63:460-468 DOI: $10.1159 / 000466691$ 


\section{Current Study}

In the current study, we used a large national sample of adults to examine whether 2 measures of subjective memory ( 1 self-comparison and 1 age-anchored comparison) are differentially related to aspects of psychological well-being: depressive symptoms and life satisfaction. Although much of the current literature has focused on older adults, we include middle-aged adults, based on recent work suggesting a peak in self-rated memory problems around the ages of 45-54 years [20]. There were 2 aims in the current analyses:

1 to examine age differences in self-comparison and age-anchored comparison ratings of subjective memory and

2 to examine whether self-comparisons and age-anchored comparisons were associated with depressive symptoms and life satisfaction.

\section{Subjects and Methods}

\section{Participants}

Data were collected as part of the second wave of the Midlife in the United States (MIDUS) survey (subjective memory had not been assessed as part of the first wave of the MIDUS). The participants had originally been recruited into the first wave of MIDUS (1995-1996) through random digit dialing over the 48 contiguous United States; while MIDUS includes a sample recruited from across the nation, it is not nationally representative with the requisite survey weighting for analysis. Full details of the recruitment procedure are described in Brim et al. [21]. The total sample size at the first wave was 7,108. At the second wave, 4,963 individuals returned to complete the survey in 2002-2006, representing a $69.8 \%$ retention rate [22]. At the second wave, the participants completed a 45-min phone interview and 2 self-administered questionnaire packets. The MIDUS survey complied with institutional review board standards of the University of Wisconsin, and the study protocol was approved by the human study committee.

Missing data occurred when participants failed to return packets or returned incomplete packets, reducing the available sample to 3,924 (a response rate of 79.4\%). Participants were also excluded if they did not participate in the cognitive protocol, so that the final sample for the current analyses was 3,434. Individuals providing complete data were more likely to be older $(t(4,960)=-5.79, p<$ $0.01)$, Caucasian $\left(\chi^{2}(1)=69.77, p<0.01\right)$, female $\left(\chi^{2}(1)=14.36, p\right.$ $<0.01)$, and higher in education $\left(\chi^{2}(4)=9.73, p<0.01\right)$ than those with incomplete data. The demographic characteristics for the final analytic sample appear at the bottom of Table 1 . The sample in the current analysis was $55.1 \%$ female; $53.1 \%$ had some college or lower education, $92.5 \%$ were Caucasian, and the average age was 56.1 years $(\mathrm{SD}=12.19)$.

\section{Measures}

Subjective Memory. Two items assessed subjective memory. The self-comparison subjective memory item asked individuals to compare their current memory to their memory 5 years ago using a rating scale from 1 (improved a lot) to 5 (gotten a lot worse). The participants were also asked to compare their memory to other individuals of the same age using a rating scale from 1 (excellent) to 5 (poor). To ease interpretation in the current analysis, these items were rescaled so that higher values represented better-perceived memory functioning (i.e., higher subjective memory ratings).

Psychological Well-Being: Depressive Affect and Life Satisfaction. Psychological well-being was operationalized using depressive affect and life satisfaction measures. For depressive affect, the participants completed a checklist of 8 depression-related symptoms derived from the Diagnostic and Statistical Manual of Mental Disorders [23] that included: felt sad/depressed; lost interest in most things; felt tired/low energy; lost/increased appetite; trouble sleeping; trouble concentrating; felt down/worthless; and thought a lot about death. Items were rated as yes or no, and a total score was created by summing the number of symptoms endorsed. Previous work using this measure reported good construct validity, with higher scores related to greater engagement with mental health services [24]. The participants rated their life satisfaction in 4 specific domains (work, health, relationship with spouse, and relationship with children) as well as overall [25]. Each was rated on a scale of 0 (worst possible) to 10 (best possible) and the average of the scores was computed as a measure of life satisfaction. Cronbach's $\alpha$ in the current study was 0.68 .

Covariates. Based on previous work on subjective memory and psychological well-being, we also included a number of relevant covariates: age, gender, education, ethnicity, neuroticism, and objective memory performance (see Table 1 for distributions). Previous work indicates that these variables are significantly related to both the outcomes of interest and perceptions of memory performance; hence, we accounted for their potential confounding effects [20, 26-28]. Neuroticism was assessed using a personality scale developed for the MIDUS study [29]. The participants indicated the extent to which 4 adjectives described them (moody, worrying, nervous, and calm) on a scale from 1 (not at all) to 4 (a lot). These items were then averaged to create a total neuroticism score (Cronbach's $a=0.74$ ). The participants completed 2 measures of episodic memory via telephone. These measures included an immediate recall test of 15 words and a delayed recall of the same list of words. The participants were read the list of words at a rate of 1 word per second and given $1 \mathrm{~min}$ for recall. Following a filled delay of approximately $12 \mathrm{~min}$, the participants were again given another minute for recall. Previous work indicates these scores load on 1 factor for objective memory performance [30]. The scores were standardized before inclusion in the regressions.

\section{Analytic Strategy}

Analyses were conducted in Mplus [31] in a series of steps. First, we computed correlations between all variables, including covariates, prior to conducting the primary analyses. Next, descriptive analyses explored the distributions of the 2 subjective memory variables across age.

We addressed the aims of our analyses simultaneously using structural equation modeling (SEM). SEM allowed us to evaluate the systems of equations for our aims as well as model depressive symptoms and life satisfaction as latent outcomes. Latent variables improve the precision of measurements by capitalizing on the common variance among related items. The depressive symptoms were binary variables, and full-information maximum likelihood 
Table 1. Descriptive statistics and correlations

\begin{tabular}{|c|c|c|c|c|c|c|c|c|c|c|}
\hline 1. SM age-anchored comparison & $0.42 *$ & & & & & & & & & \\
\hline 2. Age & -0.02 & -0.01 & & & & & & & & \\
\hline 3. Gender (Ref. $=$ female $)$ & $0.04^{*}$ & $0.04^{*}$ & 0.02 & & & & & & & \\
\hline 4. Education (Ref. $=$ HS degree) & 0.01 & $0.16^{*}$ & $-0.11^{*}$ & $0.10^{*}$ & & & & & & \\
\hline 7. Objective memory & 0.01 & $0.08^{*}$ & $-0.25^{*}$ & $-0.20^{*}$ & $0.16^{*}$ & $0.06^{*}$ & 0.01 & & & \\
\hline 8. Depressive symptoms & $-0.12^{*}$ & $-0.15^{*}$ & $-0.09^{*}$ & $-0.13^{*}$ & $-0.05^{*}$ & -0.01 & $0.21^{*}$ & $0.03^{*}$ & & \\
\hline 9. Life satisfaction & $0.11^{*}$ & $0.24^{*}$ & $0.13^{*}$ & -0.02 & $0.06^{*}$ & 0.02 & $-0.25^{*}$ & 0.01 & $-0.19^{*}$ & \\
\hline Mean & -0.39 & 2.52 & 56.11 & $55.13 \%$ & $25.22 \%$ & $92.49 \%$ & 2.05 & 0.04 & 0.62 & 7.78 \\
\hline $\mathrm{SD}$ & 0.70 & 0.90 & 12.19 & & & & 0.62 & 1.00 & 1.74 & 1.22 \\
\hline
\end{tabular}

${ }^{*} p<0.05$. Summary scores were used to represent depressive symptoms and life satisfaction. SM, subjective memory; HS, high school.

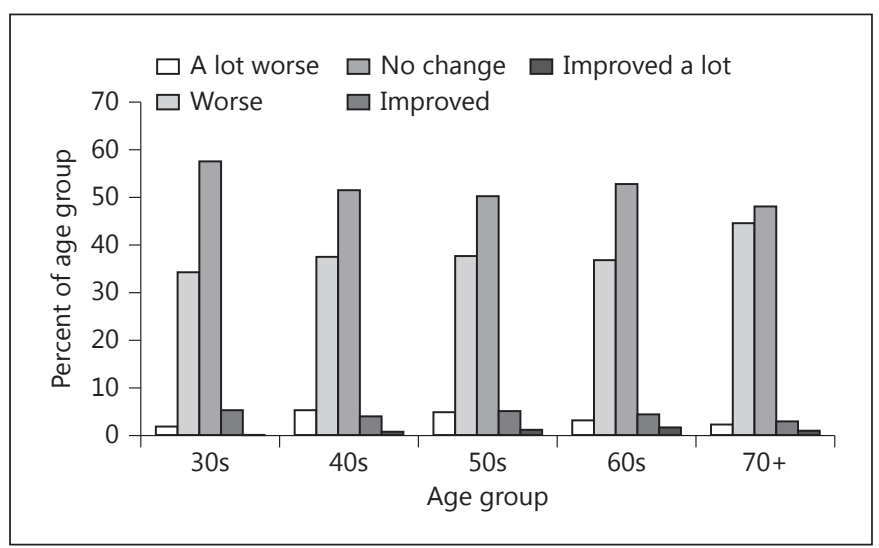

Fig. 1. Cross-sectional trends in subjective memory self-comparisons. Numbers of subjects by age group: 30 s, $n=288$; 40 s, $n=854$; 50s, $n=971 ; 60$ s, $n=764 ; 70+, n=557$.

with numerical integration was used to estimate these models. For models using this estimation method, only comparative fit indices are provided (e.g., Akaike information criterion) and model fit was established by examination of the width of the confidence intervals and the stability of the factor loadings. Standardized coefficients are reported for all paths.

Constraints were added to the model to test the equivalence of the coefficients of self- and age-anchored comparisons predicting depressive symptoms and life satisfaction. The significance of these constraints was evaluated using the Wald test [32].

Prior to conducting the SEM, we examined the multivariate distributions for outliers. Less than $3 \%$ of the observations met the

Subjective Memory in a National Sample criteria for multivariate nonnormality using the Mahalanobis distance. All analyses were conducted with and without these individuals to ensure the consistency of our results. Linearity of relationships was established using diagnostic plots of residuals.

Age was treated continuously, and linear and quadratic effects for age were tested in these models. We also included age by selfand age-anchored comparison interactions to account for age moderation of the effects of subjective memory on life satisfaction and depressive symptoms. Continuous covariates were grand mean centered, and categorical covariates were effect coded to ease interpretation of the final models. All covariates were treated as control variables and did not include interactions with any of the primary variables.

\section{Results}

The descriptive statistics and correlations for the primary analytic variables appear in Table 1 . The 2 subjective memory item ratings were moderately correlated $(r=0.42)$. Age was not significantly correlated with either measure of subjective memory. There was little variation in the magnitude of correlations between subjective memory measures and the outcomes of interest. The outcome measures were significantly intercorrelated, indicating the importance of using multivariate analysis to examine outcomes simultaneously.

For descriptive purposes, Figures 1 and 2 display the 2 subjective memory items in the current sample by age decade. Most of the adults in our sample perceived no 
Table 2. Structural equation modeling results for aims 1 and 2

\begin{tabular}{|c|c|c|c|c|}
\hline & \multicolumn{2}{|l|}{ Aim 1} & \multicolumn{2}{|l|}{$\operatorname{Aim} 2$} \\
\hline & $\begin{array}{l}\text { self- } \\
\text { comparisons }\end{array}$ & $\begin{array}{l}\text { age-anchored } \\
\text { comparisons }\end{array}$ & $\begin{array}{l}\text { life } \\
\text { satisfaction }\end{array}$ & $\begin{array}{l}\text { depressive } \\
\text { symptoms }\end{array}$ \\
\hline Age (linear) & $-0.051(0.022)^{*}$ & $0.007(0.021)$ & $0.069(0.063)$ & $-0.166(0.108)$ \\
\hline Age (quadratic) & $0.001(0.02)$ & $-0.009(0.018)$ & $0.017(0.019)$ & $-0.059(0.033)$ \\
\hline Gender (Ref. $=$ female $)$ & $-0.005(0.02)$ & $-0.021(0.019)$ & $0.055(0.018)^{*}$ & $0.176(0.029)^{*}$ \\
\hline Ethnicity (Ref. = Caucasian) & $-0.03(0.017)$ & $0.004(0.018)$ & $-0.009(0.017)$ & $0.001(0.028)$ \\
\hline Education (Ref. = HS degree) & $-0.012(0.02)$ & $0.156(0.019)^{*}$ & $0.018(0.019)$ & $-0.006(0.031)$ \\
\hline Neuroticism & $-0.21(0.019)^{*}$ & $-0.215(0.018)^{*}$ & $-0.315(0.017)^{*}$ & $0.307(0.028)^{*}$ \\
\hline Objective memory & $-0.007(0.021)$ & $0.087(0.02)^{*}$ & $0.015(0.019)$ & $0.005(0.033)$ \\
\hline Self-comparisons & & & $-0.019(0.019)$ & $-0.012(0.029)$ \\
\hline Age-anchored comparisons & & & $0.256(0.019)^{*}$ & $-0.15(0.029)^{*}$ \\
\hline Self-by-age interaction & & & $-0.007(0.023)$ & $0.024(0.041)$ \\
\hline Age-anchored-by-age interaction & & & $0.071(0.059)$ & $0.078(0.099)$ \\
\hline
\end{tabular}

Models conducted simultaneously for aims 1 and 2. Standardized coefficients reported. ${ }^{*} p<0.05$. HS, high school.

change in their memory over the target time period (the self-comparison item). As expected, there was a tendency for older adults (aged 70+ years) to endorse their memory having declined over the past 5 years. When comparing themselves to others their age (age-anchored comparison), most participants felt their memory was good, with only small proportions considering themselves to have poorer memory than others their age (0.72-2.34\%).

The SEM allowed us to simultaneously examine the aims of the current paper. The full results of this model appear in Table 2. Although many of the covariates were not significant (e.g., ethnicity), removing these did not change substantive findings and the results are reported for the full model.

Regarding our first aim (Table 2, aim 1), age was significantly related to subjective memory self-comparisons such that older age was related to lower self-comparisons, indicating a perceived decline over the last 5 years $(b=$ $-0.051, \mathrm{SE}=0.021, p=0.017)$. Age was not significantly related to age-anchored comparisons $(b=0.005, \mathrm{SE}=$ $0.020, p=0.798$ ).

For our second aim (Table 2, aim 2), age-anchored comparisons - but not self-comparisons - were significantly related to depressive symptoms (age-anchored: $b=$ $-0.150, \mathrm{SE}=0.029, p<0.001$; self-comparison: $b=-0.012$, $\mathrm{SE}=0.029, p=0.691$ ) and life satisfaction (age-anchored: $b=0.256, \mathrm{SE}=0.019, p<0.001$; self-comparison: $b=$ $-0.019, \mathrm{SE}=0.019, p=0.336$ ). Higher age-anchored comparisons were related to greater life satisfaction and lower

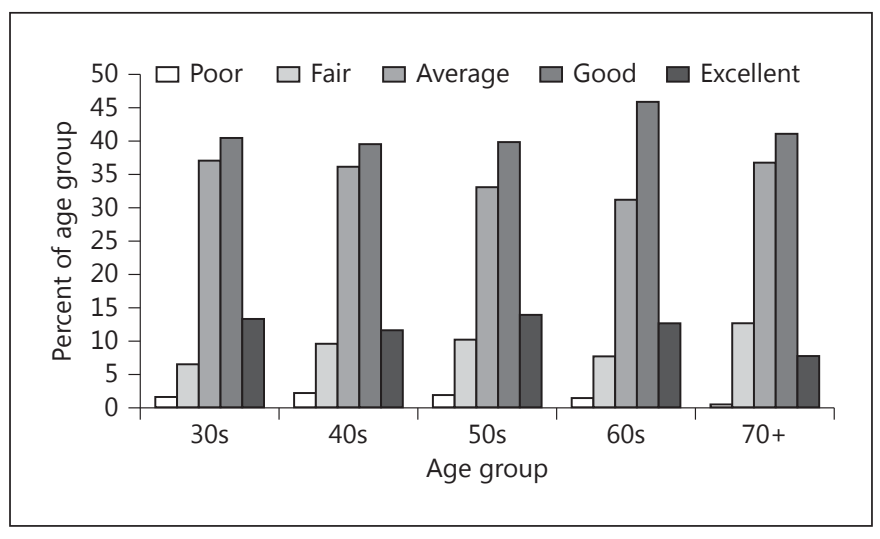

Fig. 2. Cross-sectional trends in subjective memory age-anchored comparisons. Numbers of subjects by age group: 30 s, $n=288 ; 40$ s, $n=854 ; 50 \mathrm{~s}, n=971 ; 60 \mathrm{~s}, n=764 ; 70+, n=557$.

depressive symptoms. Neither effect was moderated by age (all $p$ values $>0.234$ ).

Poor power or measurement instability could have contributed to a lack of overall significance for self-comparisons, and the Wald significance test was computed to determine whether the coefficients for self- and age-anchored comparisons were statistically equivalent. This test was significant for both outcomes (life satisfaction: Wald $(\mathrm{df}=1)=52.32, p<0.001$; depressive symptoms: Wald $(\mathrm{df}=1)=5.86, p=0.016)$, indicating that the relationship between age-anchored comparisons and the 


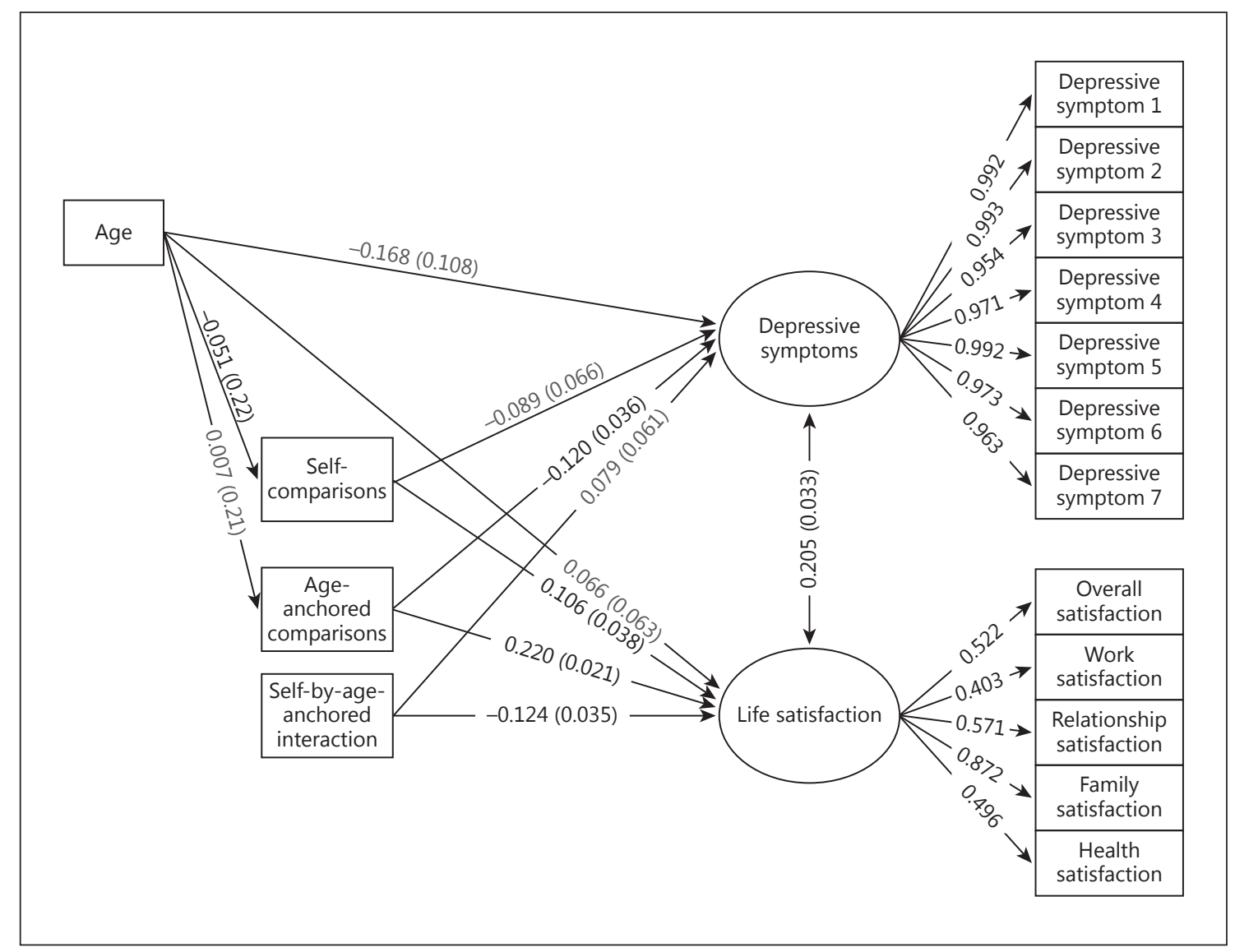

Fig. 3. Final structural equation model predicting depressive symptoms and life satisfaction.

outcomes was significantly stronger than that between self-comparisons and the outcomes.

In a supplemental analysis, we examined the interaction between self- and age-anchored comparisons. This interaction was only significant for life satisfaction $(b=$ $-0.124, \mathrm{SE}=0.035, p<0.001)$. Individuals rating themselves poorly on both self- and age-anchored comparisons had the lowest levels of life satisfaction. The final model with this interaction included appears in Figure 3.

\section{Discussion}

Our findings indicate that in this sample of adults in mid- to late life, lower self-comparison ratings only (rating current memory as worse than memory 5 years ago) were associated with older age. Higher age-anchored comparisons (rating memory as the same or better than that of one's peers) were associated with better psycho- logical well-being (lower depressive symptoms and higher life satisfaction) regardless of age. The combination of poor self- and age-anchored comparisons (individuals who perceived a decline in memory over the past 5 years and perceived their current memory functioning worse than their peers') was associated with the lowest life satisfaction scores when they occurred together. Furthermore, these effects were significant while controlling for demographic factors, neuroticism, and actual memory performance. These findings speak to the importance of multi-item subjective memory assessments for accurately capturing the nuances of the memory experience and ultimately improving their predictive utility $[33,34]$.

The potential immediate as well as long-term clinical implications of subjective memory in mid-and late life have led researchers to increasingly focus their attention on perceptions of memory in addition to memory performance, particularly throughout the aging process. However, examining the role of subjective memory - or sub- 
jective cognition more broadly - is considerably limited by a lack of commonly accepted terminology, clearly defined concepts, and consistent approaches to measurement $[7,35]$. Furthermore, within relatively consistent conceptual definitions, such as subjective memory as a measure of individual memory perceptions, a variety of measurement items are employed which rely on different levels and contexts of comparison. This study contributes a clarification of the relationships between 2 such frames of reference in ratings of subjective memory (self-comparison and age-anchored comparison) with concurrent psychological well-being.

We proposed that self-comparisons would reflect ability judgments and be more strongly related to both positive and negative aspects of psychological well-being. Instead, self-comparisons were only associated with life satisfaction, and furthermore only in the context of poorer age-anchored comparisons. This suggests that other factors may influence self-comparisons more than actual memory functioning; however, what those factors may be is unclear from the current data. One telling result is the association between neuroticism and subjective memory. It is possible that self-comparisons are more impacted by personality factors particularly when an individual is asked to retrospect over long periods of time (e.g., 5 years). An alternative is that self-comparisons capture age stereotypes rather than assessments of memory performance. Cavanaugh et al. [8] suggest that long time intervals cause the individual to access schemas related to aging rather than recall of actual performance, which is supported by the association between self-comparisons and age found in the current study. An older adult assumes that because memory is supposed to decline with age, their memory must be poorer than it was 5 years ago.

In contrast, we suggested that age-anchored comparisons would specifically relate to life satisfaction given the activations of memory concerns (rather than decrements in performance). Rating memory as the same or better than that of one's peers was associated with lower depressive symptoms and higher life satisfaction. This is consistent with a broader perspective that age-anchored comparisons activate social threat mechanisms and indicates that the impact of these mechanisms is more widespread. In addition to activating social threat, age-anchored comparisons may reflect aspects of actual performance from recent memory. Both younger and older adults may use comparisons with other individuals at or about their age as a metric of their current memory functioning. If they feel they are better than average [12], psychological wellbeing is preserved, while perceiving impairments relative to age-matched peers results in poorer psychological well-being regardless of how it is measured.

A limitation to the current study is the cross-sectional approach, which prohibits our ability to examine the temporal ordering of these relationships. Increases in depression may lead an individual to perceive memory decline rather than the alternative. Additionally, we were only able to examine the differential effects of 2 subjective memory items, although many different approaches are used within the scientific literature. However, self-comparisons versus age-anchored comparisons do represent 2 different methods of contextualizing subjective memory, and our study supports the differential relationships between these types of measures and outcomes relevant to well-being. A multi-item assessment approach is valuable for understanding an individual's perceptions of their memory, and the interaction between these items reinforces the need to treat subjective memory as a multidimensional construct. An additional limitation is the use of depressive symptoms rather than a more graded evaluation of depressive affect. In sensitivity analyses, we found that excluding individuals with more than 3 depressive symptoms resulted in the age-anchored comparisons no longer significantly relating to symptoms. However, this is likely due in part to reduced variability in depressive symptoms, as this led to the exclusion of over 100 individuals.

Future research on subjective memory should attempt to address the measurement and temporality issues discussed above. Developing brief but comprehensive assessments for inclusion in large national samples would enhance our ability to describe this construct in representative populations across the lifespan. Further, longitudinal analyses would provide the opportunity to differentiate those individuals who experience problems with memory prior to emotional and functional decrements from those who experience the opposing trajectory. Identifying these differing trajectories would elucidate the underlying processes driving perceived memory decline across important subgroups within the population. Research focusing on the specific reasons for perceived memory decline should include individuals from across the adult lifespan to understand the underlying mechanisms.

Based on our findings, the consideration of specific items used in subjective memory assessment is important beyond its implications for identifying a preclinical stage of cognitive impairment. The context of comparison, specifically in reference to one's self over time or in reference to others, demonstrates differential associations with as-
466

Gerontology 2017;63:460-468

DOI: $10.1159 / 000466691$
Mogle/Hill/McDermott 
pects of psychological well-being. This carries important implications, particularly given the well-established links between subjective memory and affective symptoms [6]. Disentangling these complex relationships is key to identifying intervention targets: poor subjective memory ratings may indicate the need for more extensive mental health screenings - or, alternatively, the introduction of cognitive strategies and supports to maintain everyday functional ability. In order to identify individually relevant targets for an intervention, we must improve our ability to assess subjective memory with more specificity. Although examining links between subjective memory and cognitive decline is an important area of research, the immediate impact of maintaining high levels of functioning in daily activities as well as positive mental health is important for long-term cognitive (and, more broadly, health and quality-of-life) outcomes.

\section{Statement of Ethics}

The MIDUS survey complied with institutional review board standards of the University of Wisconsin, and the study protocol was approved by the human study committee. This is consistent with the ethical standards of the relevant national and institutional committees on human experimentation and with the Helsinki Declaration of 1975, as revised in 2008.

\section{Disclosure Statement}

The authors have no conflicts of interest to declare.

\section{Financial Support}

This research was supported by a grant from the National Institute on Aging (P01-AG020166) to conduct a longitudinal follow-up of the MIDUS investigation; the original study was supported by the John D. and Catherine T. MacArthur Foundation Research Network on Successful Midlife Development.

\section{References}

1 Pearman A, Storandt M: Predictors of subjective memory in older adults. J Gerontol B Psychol Sci Soc Sci 2004;59:P4-P6.

2 Snitz BE, Morrow LA, Rodriguez EG, Huber KA, Saxton JA: Subjective memory complaints and concurrent memory performance in older patients of primary care providers. J Int Neuropsychol Soc 2008; 14:1004-1013.

3 Buckley R, Saling MM, Ames D, Rowe CC, Lautenschlager NT, Macaulay SL, et al: Factors affecting subjective memory complaints in the AIBL aging study: biomarkers, memory, affect, and age. Int Psychogeriatr 2013;25: 1307-1315.

4 Chin J, Oh KJ, Seo SW, Na DL: Are depressive symptomatology and self-focused attention associated with subjective memory impairment in older adults? Int Psychogeriatr 2014; 26:573-578.

5 Minett TSC, Da Silva RV, Ortiz KZ, Bertolucci PHF: Subjective memory complaints in an elderly sample: a cross-sectional study. Int J Geriatr Psychiatry 2008;23:49-54.

6 Hill NL, Mogle J, Wion R, Munoz E, DePasquale N, Yevchak AM, et al: Subjective cognitive impairment and affective symptoms: a systematic review. Gerontologist 2016;56:e109-e127.

7 Rabin LA, Smart CM, Crane PK, Amariglio RE, Berman LM, Boada M, et al: Subjective cognitive decline in older adults: an overview of self-report measures used across 19 international research studies. J Alzheimers Dis 2015;48(suppl 1):S63-S86.
8 Cavanaugh JC, Feldman JM, Hertzog C: Memory beliefs as social cognition: a reconceptualization of what memory questionnaires assess. Rev Gen Psychol 1998;2:48-65.

9 Tandetnik C, Farrell MT, Cary MS, Cines S, Emrani S, Karlawish J, et al: Ascertaining subjective cognitive decline: a comparison of approaches and evidence for using an ageanchored reference group. J Alzheimers Dis 2015;48(suppl 1):S43-S55.

10 Suls J, Wheeler L: Social comparison theory; in Van Lange PAM, Kruglanski AW, Higgins ET (eds): Handbook of Theories of Social Psychology. London, SAGE, 2012, vol 1, chapter 22, pp 460-482.

11 Fastame MC, Penna MP, Rossetti ES, Agus M: Perceived well-being and metacognitive efficiency in life course: a developmental perspective. Res Aging 2013;35:736-749.

12 Alicke MD: Evaluating social comparison targets; in Suls J, Wheeler L (eds): Handbook of Social Comparison: Theory and Research. New York, Springer, 2000, pp 271-293.

13 Huppert FA: Psychological well-being: evidence regarding its causes and consequences. Appl Psychol Health Wellbeing 2009;1:137164

14 Metternich B, Schmidtke K, Hüll M: How are memory complaints in functional memory disorder related to measures of affect, metamemory and cognition? J Psychosom Res 2009;66:435-444.

15 Zuniga KE, Mackenzie MJ, Kramer A, McAuley E: Subjective memory impairment and well-being in community-dwelling older adults. Psychogeriatrics 2016;16:20-26.
16 Kessler E-M, Bowen CE, Baer M, Froelich L, Wahl H-W: Dementia worry: a psychological examination of an unexplored phenomenon. Eur J Ageing 2012;9:275-284.

17 Mol ME, Ruiter RA, Verhey FR, Dijkstra J, Jolles J: A study into the psychosocial determinants of perceived forgetfulness: implications for future interventions. Aging Ment Health 2008; 12:167-176.

18 Toffalini E, Borella E, Cornoldi C, De Beni R: The relevance of memory sensitivity for psychological well-being in aging. Qual Life Res 2016;25:1943-1948.

19 Kotter-Grühn D, Hess TM: The impact of age stereotypes on self-perceptions of aging across the adult lifespan. J Gerontol B Psychol Sci Soc Sci 2012;67:563-571.

20 Begum A, Dewey M, Hassiotis A, Prince M, Wessely S, Stewart R: Subjective cognitive complaints across the adult life span: a 14year analysis of trends and associations using the 1993, 2000 and 2007 English Psychiatric Morbidity Surveys. Psychol Med 2014;44: 1977-1987.

21 Brim OG, Ryff CD, Kessler RC: How healthy are we? A national study of well-being at midlife. University of Chicago Press, 2004. https://books.google.com/books?hl= en\&lr=\&id=tZKRJsi_-VoC\&oi=fnd\&pg=PA1 $\& \mathrm{dq}=$ Brim, + Ryff, + and + Kessler $+(2004) \&$ ots $=$ 5x3P4sKbVO\&sig=KVYlk5QoNhyqsKq7vyS YDBZ_fug (accessed Dec 19, 2016).

22 Radler BT, Ryff CD: Who participates? Accounting for longitudinal retention in the MIDUS national study of health and well-being. J Aging Health 2010;22:307-331. 
23 American Psychiatric Association: Diagnostic and Statistical Manual of Mental Disorders, revised (DSM-III-R). Washington, American Psychiatric Association, 1987.

24 Wang PS, Berglund P, Kessler RC: Recent care of common mental disorders in the United States: prevalence and conformance with evidence-based recommendations. J Gen Intern Med 2000;15:284-292.

25 Prenda KM, Lachman ME: Planning for the future: a life management strategy for increasing control and life satisfaction in adulthood. Psychol Aging 2001;16:206-216.

26 Ficker LJ, Lysack CL, Hanna M, Lichtenberg PA: Perceived cognitive impairment among African American elders: health and functional impairments in daily life. Aging Ment Health 2014;18:471-480.

27 Lehrner J, Moser D, Klug S, Gleiß A, Auff E, Dal-Bianco P, et al: Subjective memory complaints, depressive symptoms and cognition in patients attending a memory outpatient clinic. Int Psychogeriatr 2014;26:463-473.
28 Snitz BE, Yu L, Crane PK, Chang C-CH, Hughes TF, Ganguli M: Subjective cognitive complaints of older adults at the population level: an item response theory analysis. Alzheimer Dis Assoc Disord 2012;26:344-351.

29 Lachman ME, Weaver SL: The Midlife Development Inventory (MIDI) personality scales: scale construction and scoring; technical report, Brandeis University, Waltham, 1997.

30 Tun PA, Lachman ME: Telephone assessment of cognitive function in adulthood: the Brief Test of Adult Cognition by Telephone. Age Ageing 2006;35:629-632.

31 Muthén LK, Muthén BO: Mplus: Statistical Analysis with Latent Variables: User's Guide. Los Angeles, Muthén \& Muthén, 2010.
32 Harrell F: Regression Modeling Strategies With Applications to Linear Models, Logistic Regression, and Survival Analysis. New York, Springer, 2001.

33 Amariglio RE, Townsend MK, Grodstein F, Sperling RA, Rentz DM: Specific subjective memory complaints in older persons may indicate poor cognitive function. J Am Geriatr Soc 2011;59:1612-1617.

34 Laske C, Sohrabi HR, Jasielec MS, Müller S, Koehler NK, Gräber S, et al: Diagnostic value of subjective memory complaints assessed with a single item in dominantly inherited Alzheimer's disease: results of the DIAN study. Biomed Res Int 2015;2015:828120.

35 Jessen F, Amariglio RE, van Boxtel M, Breteler M, Ceccaldi M, Chételat G, et al: A conceptual framework for research on subjective cognitive decline in preclinical Alzheimer's disease. Alzheimers Dement 2014;10:844852 